\title{
Formação em métodos de pesquisa na pós-graduação: abordagens multimétodos para as demandas da atualidade
}

\section{Training in Research Methods in Graduate Education: Multi-Methods Approaches for Today's Research Needs}

\author{
Salvador A. M. Sandoval ${ }^{*}$
}

\begin{abstract}
RESUMO
$\mathrm{O}$ artigo discute a importância de ensinar métodos de pesquisa qualitativos e quantitativos como abordagens complementares conforme os objetivos do pesquisador. Argumenta-se que hoje o conhecimento científico não é apenas objeto de universidades, e que o mundo acadêmico mais que conhecimento científico, desde o século 19, faz parte do processo de formulação de políticas públicas, e que no século 20 o conhecimento científico se tornou um elemento-chave na delimitação de políticas. Por esse motivo, há um importante desenvolvimento das abordagens quantitativas, justamente por poderem fazer generalizações sobre uma população ou um segmento populacional, objeto de uma política, uma vez que políticas públicas visam atender às coletividades para atender a necessidade de obter dados científicos sobre essas coletividades. O artigo mostra a dupla tendência na história dos métodos de pesquisa social: por um lado o interesse meramente acadêmico e por outro a necessidade de governantes de obterem dados empíricos sobre os povos por eles governados. Essa dupla trajetória dos métodos de pesquisa social vai se integrar no século 20, entre as Primeira e Segunda Guerras Mundiais, quando governantes olham para as universidades para formar os pesquisadores aplicados nas metodologias de pesquisa, adequadas para realizar pesquisas como subsídios à formulação e avaliação de política pública.

Palavras-chave: Metodologia da Pesquisa. Métodos de Pesquisa. Pesquisa e Política Pública. História dos Métodos de Pesquisa.
\end{abstract}

* Pontifícia Universidade Católica de São Paulo Programa de Pós-Graduação em Psicologia Social. São Paulo, São Paulo, Brasil. E-mail: sams1910@gmail.com. https://orcid.org/0000-00030954-3741. 


\begin{abstract}
The article discusses the importance of teaching qualitative and quantitative research methods as complementary approaches depending on the objectives of the researcher. We argue that today scientific knowledge is not just a object of universities and the academic world but rather scientific knowledge since the 19th Century is part of a process of formulating public policies and in the 20th Century scientific knowledge becomes a key element in the delimitation of policies and for this reason there is an important development in the quantitative approaches precisely in order to be able to generalize findings to a population or population segment, the objective of policy, since the intent of public policy is to reach collectivities and thus the need for scientific data on those collectivities. This article shows the double tendency in the history of social research methods: on the one hand, the mere interest in academics and on the other hand, the needs of governments to have empirical data about their peoples. This double trajectory of social research methods will become integrated in the 20th Century between the First and Second World Wars when governments look to universities to train applied researchers in research methodologies more appropriate for studies that provide inputs to the formulation and evaluation of public policy.

Keywords: Research methodology. Research methods. Research and Public Policy. History of Research Methods.
\end{abstract}

Nos últimos vinte anos houve grandes transformações no uso e demanda para o conhecimento gerado pelas pesquisas sociais empíricas. Agências governamentais nas áreas de educação, assistência social, saúde e trabalho têm progressivamente fundamentado os parâmetros de políticas públicas com o apoio das pesquisas e levantamentos empíricos. Assim, observamos a mesma tendência nos setores não governamentais, como nas áreas de partidos públicos, sindicatos e órgão de lobby para políticas públicas. Ao mesmo tempo, estamos assistindo a um movimento incipiente que pretende avaliar o desempenho dos serviços de assistência pública, das políticas sociais e das pessoas nos governos, sob uma perspectiva dos usuários/cidadãos.

Essas mudanças põem em discussão a problemática da formação em métodos de pesquisa, no contexto da pós-graduação, em que um grande número de futuros administradores de nossa sociedade é formado. Com uma tradição de pesquisa historiográfica e qualitativa, as pós-graduações em ciências humanas, no tocante à formação em metodologias de pesquisa empírica, estão confrontadas com a necessidade de repensar os conteúdos das disciplinas de metodologia diante das novas exigências de conhecimento científico para o mundo do setor público e do mercado que apresentam hoje. 
Os debates mais acalorados de hoje sobre a criação, mudança ou eliminação de qualquer política pública se fundamentam em estudos e dados empíricos, que são usados pelas partes para justificar e debater suas pretensões e rejeitar posições contrárias aos seus interesses. As sociedades atuais são movidas por esses debates, com base em informações e conhecimentos científicos produzidos por pesquisadores inseridos em universidades, institutos públicos, e centros particulares de pesquisa, que realizam estudos atendendo a diversos interesses políticos e sociais nas sociedades, com o intuito de influenciar a formulação de políticas públicas e sociais de governos. Para tanto, a pesquisa hoje assume um papel central nas discussões sobre os rumos da intervenção pública na vida social e seus impactos nos cidadãos, alvos dessas políticas.

Pensando no papel central que a pesquisa científica vem assumindo na sociedade moderna, neste artigo pretendemos discutir os métodos de coleta de dados no caso de pesquisas empíricas, que estudam fenômenos sociais. Parte-se da suposição de que há, sistematicamente, que coletar dados empíricos sobre a realidade a ser pesquisada, e que há para essa finalidade uma diversidade de métodos de coleta e registro de dados sociais, conforme os objetivos e interesses do pesquisador e a sua finalidade de retratar essa realidade.

No entanto, para discutir a problemática do ensino da metodologia de pesquisa nas Ciências Humanas no Brasil, se faz necessário lembrar que pesquisas neste campo tiveram o seu início acontecendo junto aos interesses acadêmicos das universidades e à necessidade de governantes obterem dados sobre os povos que governavam, com o intuito de definir políticas públicas.

\section{Início da Pesquisa Social Empírica}

Esse enfoque de estudar empiricamente os acontecimentos sociais teve seu início no século 18, quando estudiosos europeus se aventuraram a pesquisar sociedades e culturas, então desconhecidas e notavelmente diferentes das europeias. Diante dos desafios de estudar sociedades e culturas tão distintas, encontradas na África e nas Américas, aparecem os primeiros estudos usando um procedimento que viria a ser conhecido como "etnografia", desenvolvido por pesquisadores alemães, Gerhard Friedrich Mueller (1733-43) e logo depois por Johann Friedrich Schöpperlin e A. F. Thilo em 1767. Nesse contexto histórico de pesquisa social, os enfoques da emergente antropologia das culturas e sociedades tinha uma clara fundamentação positivista, tanto que se poderia dizer que a origem da etnografia estava definitivamente dentro de uma perspectiva positivista de ciência do homem daquela época (VERMEULEN, 2008). 
No decorrer do século 19, entrando no século 20, o uso e aperfeiçoamento do método etnográfico se deu com a constituição da disciplina de Antropologia graças às contribuições de importantes pesquisadores ocidentais como E.B. Tylor (1832-1917) do Reino Unido, Lewis H. Morgan (1818-1881), americano, Franz Boas (1858-1942), Bronislaw Malinowski (1858-1942), Ruth Benedict e Margaret Mead (1901-1978) que, em conjunto, foram responsáveis pela consolidação da disciplina da Antropologia, salientando que a etnografia se tornou o método de coleta de dados empíricos predileto nos estudos de sociedades e suas culturas (GULLION, 2016).

Ao mesmo tempo, nos contextos europeus e norte-americanos, a pesquisa estatística demográfica e social teve seu início no século 19 impulsionada, não por um objetivo acadêmico, mas pelas necessidades de governantes de gerar conhecimento por meio de dados empíricos, para melhor formular ações e políticas públicas diante de problemas coletivos, como saúde e pobreza nos centros urbanos e fábricas, nos países em processos de industrialização.

Os censos demográficos populacionais, no sentido moderno do conceito como um levantamento sistemático e regular de toda a população de uma nação, só tiveram seus inícios no final do século 18 (primeiro na Suécia em 1747 e desde 1775 a cada cinco anos até hoje; depois nos Estados Unidos, Inglaterra e França em 1801 e, desde então, se realiza o censo nacional a cada dez anos). Desde esses inícios, o censo demográfico, como uma pesquisa regular de condições da população de um país, se tornou uma atividade de pesquisa fomentada por todos os governos nacionais modernos.

Tal foi o caso do governo e parlamento britânico que no início do século 19 encomendou levantamentos específicos das condições de vida e de saúde de moradores de bairros pobres e proletários de Londres. Semelhantemente foram realizados vários estudos em Paris, Berlim, Nova York e Washington com finalidade de conhecer as condições de moradia, saúde, emprego e educação de residentes de bairros pobres.

Esses estudos tiveram como base metodológica os procedimentos usados nos levantamentos censitários nacionais. Os levantamentos focando apenas em populações pobres costumavam ser caros porque requeriam uma abordagem censitária de amostras grandes e, portanto, relativamente infrequentes.

Graças a essas pesquisas sociodemográficas e especialmente àquelas focadas nas condições de vida das classes proletárias inglesas, foi possível que Friedrich Engels baseasse seu estudo clássico, de 1845, na situação da classe operária na Inglaterra. Usando dados coletados pelo bureau de pesquisa do censo ou pelas comissões parlamentares, Engels desenvolveu uma análise sobre a natureza da luta de classes, assim como se manifestavam nas condições de vida do proletariado inglês. Nesse livro clássico de análise sociopolítica empí- 
rica, Engels soube usar os dados coletados nas pesquisas encomendadas pelo parlamento para demonstrar as condições das classes proletárias inglesas como consequência da exploração capitalista (ENGELS, 1969 (1845).

Uma joia de historiografia dos métodos de pesquisa social é o questionário escrito por Karl Marx, em 1880, a pedido de uma publicação francesa de esquerda, elaborado a pedido dos editores da Revue Socialiste. O questionário para pesquisar o proletariado só foi conhecido por estudiosos do Marxismo cinquenta anos depois, quando publicado no New International. Os editores da revista francesa Revue Socialiste tinham como intenção distribuir aos trabalhadores leitores franceses o questionário, com a finalidade de traçar um perfil político, socioeconômico e de condições de trabalho da classe operária em Paris. O questionário contém 101 perguntas sobre vários temas e aparentemente foi distribuído aos leitores, porém, não há registro de qualquer informação sobre a devolução dos resultados dessa primeira enquete por correspondência (NEW INTERNATIONAL, 1938).

O "questionário de Karl Marx" provavelmente teve uma baixa taxa de retorno, entendida hoje como uma das desvantagens de pesquisas feitas por correio ou por meio de assinantes. Esse baixo retorno poderia explicar a falta de registros sobre o resultado da pesquisa. O certo é que para Marx e colaboradores, levantamentos feitos por meio de questionários não apresentavam nenhum problema epistemológico ou destoante dos enfoques teóricos ou ideológicos dos autores, nem em termos da validade e utilidade dos dados quantitativos obtidos por esses métodos de survey. Pelo contrário, a contribuição da obra de Engels de 1845, A Situação da Classe Operária Inglesa, demonstra como um bom analista, firme em seu enfoque teórico-metodológico, pode aproveitar dados obtidos por outros pesquisadores para argumentar suas teses sem grandes obstáculos epistemológicos. Também ilustra os inícios, no século 19, sobre o uso de enquetes como forma de pesquisa social, que eram utilizadas por autoridades públicas interessadas em obter dados de populações e de segmentos populacionais com a finalidade de considerá-los na formulação de políticas públicas.

\section{Avanços nas Técnicas de Pesquisa no século 20}

Foi na primeira metade do século 20 que houve importantes avanços no desenvolvimento de técnicas de coleta de dados, tanto nas modalidades qualitativa como quantitativa. Entrevistas em profundidade representam uma das estratégias mais antigas de coleta de dados nas pesquisas sociais. Frederick 
LePlay foi talvez o primeiro pesquisador a usar esse método, no século 19, no seu estudo de famílias de classe trabalhadora (LePLAY, 1855).

No entanto, foi o trabalho do grupo de pesquisadores sociais (sociólogos, psicólogos sociais e filósofos) que hoje são conhecidos como a Escola de Chicago, da Universidade de Chicago, que inovaram no desenvolvimento de métodos de pesquisa de campo, nos seus estudos com imigrantes europeus nos bairros pobres da cidade de Chicago. Em primeiro lugar, pesquisadores da Escola de Chicago adaptaram a metodologia de etnografia usada pelos antropólogos do século 18 para conhecer as sociedades africanas e norte-americanas, para o contexto das pequenas sociedades de imigrantes camponeses, localizados no meio dos centros urbanos de Chicago e Nova York. A fim de pesquisar esses novos imigrantes, os pesquisadores adaptaram o método de etnografia clássica para estudar contextos urbanos de populações ocidentais, mantendo os traços principais da etnografia clássica sem a exigência de permanência continua do pesquisador no local. Por estar pesquisando bairros na mesma cidade, ocupados por imigrantes europeus, entendeu-se que o pesquisador poderia substituir frequentes e regulares visitas aos bairros, em lugar dos longos períodos de permanência que antropólogos do século 19 faziam para melhor conhecer a vida cotidiana e estruturas relacionais das sociedades africanas. Ao mesmo tempo, as técnicas de entrevistas informais, observação e entrevistas de história de vida seriam suficientes para a coleta de dados pertinentes à pesquisa.

The Polish Peasant in Europe and America, de Thomas e Znaniecki, em 1918, é a obra mais conhecida da Escola de Chicago em termos de suas inovações metodológicas. Além de utilizar uma etnografia adaptada para contextos urbanos, os pesquisadores também desenvolveram técnicas de entrevista de historial oral, entrevistas em profundidade e observação sistemática de relações sociais. $\mathrm{Na}$ fase de interpretação dos dados, a equipe foi pioneira em formular abordagens de análise de conteúdo que permitissem correlacionar todos dos dados obtidos pelas várias técnicas usadas.

Além das grandes contribuições teóricas nas áreas da Sociologia, Psicologia Social, Filosofia e Educação, os trabalhos da Escola de Chicago foram trabalhos seminais, metodologicamente aportando para os campos das ciências sociais (sociologia, psicologia social, educação, etc.) abordagens metodológicas de coleta e análise de dados empíricos. O fato de a Escola de Chicago ter focado em populações camponesas imigrantes na cidade de Chicago impôs a necessidade de abordar o fenômeno desconhecido em uma perspectiva qualitativa. Por essas razões, essa oportunidade de pesquisa teve com resultado uma contribuição por meio da elaboração de um conjunto de métodos de pesquisa empírica, formas de análises desses conteúdos multifacetados. 
No mesmo período em que os membros da Escola de Chicago inovaram na formulação de métodos qualitativos, vários pesquisadores em universidades americanas desenvolveram as ferramentas para viabilizar a pesquisa survey, como uma metodologia viável para pesquisas sociais. Vale mencionar que, nas décadas de 1920 e 1930, alguns cientistas desenvolveram técnicas de mensuração de atitudes, valores e crenças por meio de perguntas estruturadas a serem usadas em questionários. A invenção da mensuração por meio de escala atitudinal, a correlação de perguntas e as técnicas de formulação de respostas para conjuntos de perguntas a fim de mensurar algumas dimensões atitudinais. Desses pioneiros podemos destacar os seguintes pesquisadores que tanto contribuíram com o desenvolvimento de técnicas de elaboração de questionários: R. Likert (1932); Thurstone (1928); E.S. Bogardus (1925); Gutman (Stouffer, 1949); Osgood, (1957).

Além da invenção de uma tecnologia de formulação de perguntas para questionários, a pesquisa com survey só se torna viável como método de pesquisa social quando é possível reduzir os custos associados com pesquisas tipo censo demográfico com base em levantamentos de grande número de entrevistas. Os trabalhos de estatísticos escandinavos e holandeses, nas décadas de 1890 a 1920, vão resultar em dois métodos delimitadores de amostras de populações que se aproximam em representatividade da população maior. No final da década de 1890, Anders Kiaer (1838-1919), fundador e primeiro diretor do instituto de estatística da Noruega, Statistics Norway, foi o pioneiro no método de amostragem aleatória que hoje é usado por institutos oficiais do censo, para acompanhar segmentos específicos da população durante a década entre censos. Esse método de amostragem, chamado amostra representativa, consiste em selecionar aleatoriamente números iguais de casos extraídos de dois segmentos previamente delimitados dos dados do censo demográfico. Por meio de uma amostra aleatória de casos dos diferentes segmentos dos censos, foi obtida uma amostra representativa da população, o que possibilitou análises de um número menor de casos do que do censo demográfico. Kiaer foi o primeiro a usar uma amostra aleatória para pesquisa um programa de seguro de saúde e aposentadoria na Noruega (BETHLEHEM, 2009). Hoje podemos encontrar essa sistemática de amostragem usada pelo IBGE na sua Pesquisa Nacional por Amostra de Domicílios Contínua Mensal, que permite acompanhar os domicílios brasileiros ao longo da década com a finalidade de registrar as mudanças socioeconômicas nos anos entre os censos demográficos nacionais.

A ideia de Anders Kiaer fez com que estatísticos na Holanda pesquisassem as possiblidades de amostras menores, o que resultou no achado de intervalos de confiança que determinariam diferentes tamanhos de amostras. Por meio de práticas de amostragens na agricultura, com variados tamanhos de amostras, 
esses estatísticos poderiam determinar intervalos de confiabilidade de amostragem segundo tamanho da amostra escolhida com base na noção de escolha aleatória para aproximarem-se de uma representação de população em questão (BETHLEHEM, 2009).

Assim inicia-se uma nova fase de pesquisa survey com amostragens de diferentes tamanhos, porém também com as condições de estimar suas respectivas representatividades, conforme o tamanho da população. A possibilidade de poder estudar fenômenos sociais com amostras bastante menores do que o total da população, tornou o levantamento survey com amostragens aleatórias um método de pesquisa social usado para estudar grandes coletividades, com níveis altos de representatividade.

\section{Pesquisa Científica entre a Academia e as Políticas Públicas}

Como apontamos brevemente, o desenvolvimento dos métodos de coleta de dados sociais desde o século 19, foi determinado por duas forças que, às vezes são aparentemente contrárias. Por um lado, os métodos surgem como consequência de pesquisadores acadêmicos que registram informações dos sujeitos e coletivos sendo pesquisados, como atividade regular da investigação acadêmica que visa à aquisição de novos conhecimentos sobre o mundo. Por outro lado, no século 19, surge a necessidade de governos e organizações da sociedade adquirirem informações sobre as populações e as condições de vida que essas populações têm com a finalidade de subsidiar a formulação de políticas sociais, para responder às condições de segmentos populacionais (trabalhadores, crianças, mulheres, doentes, etc.) e ligadas aos grupos políticos organizados que pretendem representar esses segmentos na sociedade.

Assim sendo, emergem um conjunto de trabalhos desenvolvendo métodos de registro de dados de coletividades maiores, e não apenas de indivíduos ou pequenos grupos, como no caso das pesquisas acadêmicas da época, com o intuito de prover informações empíricas sobre problemas sociais suscetíveis a serem atendidos por meio de políticas públicas.

Essa dinâmica entre pesquisas com fins acadêmicos e pesquisas com fins institucionais revela uma realidade que, analiticamente, poderia ser distinguida em três níveis de análise e de coleta de dados: o nível macrossocial que se refere à sociedade como um todo; o nível mesossocial que se refere a instâncias intermediárias entre o macrossocial e a realidade no plano individual e pequenos grupos; e o nível microssocial que se refere a aquele plano dos indivíduos e seus pequenos agrupamentos como famílias, pares, etc. 
As diferenças analíticas entre os níveis de análise também foram refletidas nos métodos de coleta de dados sociais desenvolvidos ao longo dos séculos 19 e 20. Assim encontraremos métodos de coleta de dados focados em relatos de indivíduos, narrando fatos sobre eles mesmos e/ou dos pequenos grupos que compõem suas realidades cotidianas, como no caso de entrevistas em profundidade e temáticas, histórias de vida, entrevistas clínicas e testes psicológicos. Também apareceram técnicas de registro de dados sociais focando principalmente em fatos referentes aos indivíduos, inseridos de grupos e pequenas comunidades, em que o foco do registro é sobre fatos dessas pequenas comunidades, organizações, bairros, etc. como no caso de etnografias urbanas, observações participantes, grupos focais, historiais orais. E, por fim, encontramos métodos que, partindo dos modelos de levantamentos do censo, proporcionaram que pesquisadores experimentassem formas de fazer enquetes sem a necessidade de amostras universais e com instrumentos de registro de dados capazes de captar maiores nuances psicológicas nas respostas dos entrevistados.

Os três níveis de análise do social pressupõem a intencionalidade do pesquisador de focar algum nível em sua coleta de dados empíricos; entendendo que o focar em um nível reduzia a abrangência empírica adequada para simultaneamente analisar com o mesmo rigor outros níveis de análise. Dessa forma, a prática de pesquisar de modo empírico a realidade social foi tornando-se de forma conceitual mais demarcada analiticamente tanto no contexto da academia como no mundo da formulação das políticas públicas. Não poderia ser de outra maneira. A complexidade do mundo e a dinâmica social só poderiam suscitar a necessidade de desenvolver técnicas que melhor registrassem o fato empírico social, conforme os respectivos níveis analíticos da realidade social.

Diante de uma realidade social, complexa em suas estruturas dinâmicas e significados, o pesquisador é mediador entre aquela faceta da realidade social que pretende pesquisar e o método ou métodos de coleta de dados que serão necessários para um apanhado suficiente, um retrato, da fatia da realidade que o pesquisador delimitou como seu foco de pesquisa. Em meio às diversidades de métodos de registro de fatos sociais, o pesquisador enfrenta a tarefa criativa de formular uma abordagem metodológica mais adequada para o fenômeno social que investiga. Essa abordagem requer conhecimentos dos métodos disponíveis para melhor escolher aquele mix que melhor atende às expectativas da pesquisa em questão. A elaboração do plano metodológico não é aleatória nem a priori, mas a consequência da natureza empírica e teórica daquela fatia da realidade social a ser investigada. Não há método universal de coleta e registro de dados sociais, por isso há várias modalidades de métodos nas ciências humanas. 


\section{As Ciências Sociais e as Múltiplas Metodologias de Pesquisa}

$\mathrm{O}$ argumento de que diferentes métodos de coleta e registro de dados empíricos correspondem a diferentes enfoques sobre o que pesquisar está ilustrado no seguinte Diagrama 1. Neste estão distribuídos os distintos métodos de pesquisa social entre focos principais no indivíduo ou no coletivo, por um lado, e entre aqueles métodos qualitativos e quantitativos, por outro. Como podemos ver, há métodos qualitativos e quantitativos que acessam dados focados nos indivíduos, assim como vemos que há métodos tanto quantitativos como qualitativos que priorizam focos sobre o coletivo e menos no individual.

\section{DIAGRAMA 1 - MÉTODOS DE COLETA DE DADOS SOCIAIS SEGUNDO FOCO PRIORITÁRIO DO PESQUISADOR}

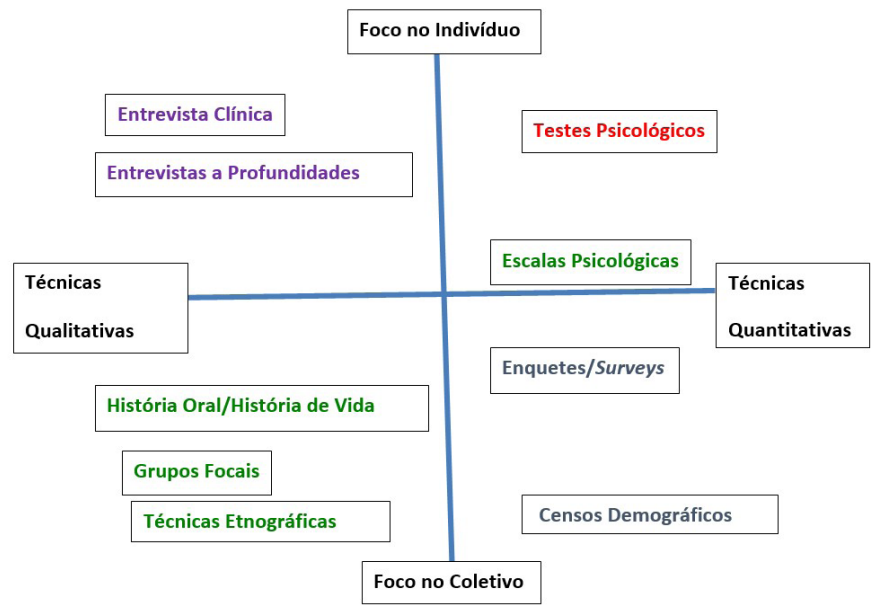

FONTE: Salvador A.M. Sandoval, 2018.

Da perspectiva dos usos das pesquisas sociais, como visto acima, desde os séculos 18 e 19, a pesquisa social foi fornecendo subsídios para a formulação de políticas públicas. Definitivamente, iniciando no século 19 e acentuando-se no século 20, as universidades passaram a ter um maior papel no desenvolvimento de pesquisas com a finalidade de subsidiar o processo de formulação de políticas públicas. No início do século 20, Max Weber apontou para esse fato ao observar como o papel da ciência na universidade era progressivamente mais procurado pelas autoridades públicas e como isso iria transformar a universidade da épo- 
ca (WEBER, 2005). Assim como previsto por Max Weber, a universidade da atualidade é vista com uma instituição de produção de conhecimento científico que poderá vir a ser uma parte essencial do processo de projetar e planejar o desenvolvimento da sociedade.

Ao mesmo tempo, notamos que governos por sua vez também se dispõem a criar institutos autônomos das universidades, para produzir o conhecimento científico que precisam para governar, quando as instituições de ensino superior nãos são capazes de atender a essa demanda. Com isso, a universidade se enfraquece como locus de produção científica socialmente relevante na sociedade.

Conforme analisamos acima, o processo de uma crescente demanda de informação científica, por parte dos planejadores de políticas públicas, resultou na elaboração de diferentes métodos de coleta de dados, com o intuito de melhor apreender dados sobre fenômenos sociais de interesse para política governamental. Em meados do século 20, isso foi reforçado pelo interesse de organizações privadas, tanto comerciais como cívicas, em também coletar dados sociais sobre segmentos sociais dentro da sociedade, com a finalidade de elaborar ações específicas para segmentos coletivos.

Assim sendo, também podemos pensar os diferentes métodos de coleta de dados com base na perspectiva do uso de conhecimentos científico para políticas públicas que, por definição, tem abrangências coletivas e, portanto, precisam de dados sociais sobre segmentos populacionais e não apenas individuais. Política pública não se formula apenas com dados científicos de casos isolados de indivíduos, visto que o foco da política pública são as coletividades na sociedade e, para tanto, a discussão sobre os rumos de política pública pressupõe informação sistemática e representativa dessas coletividades às quais seria destinada essa política.

Portanto, podemos pensar os diferentes métodos de pesquisa não apenas nas suas formas qualitativas ou quantitativas, mas no sentido de compreender que tipo de conhecimento científico é proporcionado via cada método de pesquisa, em termos de possiblidades de generalização, seja no sentido de compreender o fenômeno social ou em termos de captar a sua frequência em um segmento populacional.

O Diagrama 2, a seguir, distribui os diferentes métodos de pesquisa empírica em torno de dois vetores: um vetor sobre o grau de aprofundamento de conhecimento sobre processos sociais, e o outro vetor sobre o grau de representatividade dos achados de uma pesquisa, de uma população específica. No vetor relativo ao grau de aprofundamento de conhecimento de processos sociais, podemos ver que experimentos e métodos clínicos tendem a ser mais altos em grau de conhecimento; enquanto que entrevistas narrativas de um sujeito, ou 
entrevistas acidentais de um sujeito, seriam mais baixos. Ambos teriam baixa possiblidade de serem generalizados para alguma população.

Por outro lado, o censo demográfico é um método de pesquisa que é altamente representativo da população, porém seu grau de aprofundamento de conhecimento dessa população é bastante baixo justamente porque, ao atingir quase $100 \%$ da população, o censo demográfico sacrifica o aprofundamento das informações a favor de maior abrangência. No entanto, diferentes formas de enquetes eleitorais, de opinião e de uso de escalas psicológicas, atingem médios e altos graus de aprofundamento, mas com menor grau de representatividade em razão do uso de amostragens aleatórias em lugar de cobertura censitária.

Como se pode ver no Diagrama 2, métodos intermediários entre aqueles fundamentados em estudos de casos únicos e aqueles com base em grandes amostras, como são os métodos da etnografia, história oral, grupos focais, são métodos qualitativos que pretendem obter graus maiores de representatividade usando formas de entrevistas ainda mais abertas e menos dirigidas, que aquelas usadas nas enquetes de opinião, escalas psicológicas ou eleitorais. Esses conjuntos de métodos focalizam não no indivíduo e, sim, em algum tipo de coletividade. Dessa forma, tentam atingir essa maior representatividade por meio dessa coletividade, sem deixar de usar formas abertas de obtenção de dados narrativos.

$\begin{aligned} \text { DIAGRAMA 2: } & \text { ABORDAGENS QUALITATIVAS E QUANTITATIVAS DE } \\ & \text { PESQUISA CONFORME O NÍVEL DE APROFUNDAMENTO } \\ & \text { DOFENÔMENO E REPRESENTATIVIDADE NAPOPULAÇÃO }\end{aligned}$

Aprofundar

Processos

Alto

Experimentos

Etnografia

Pe

Pesquisa Clínica

História de Vida

Grupo Focal

Observação Participante

Entrevistas Semiabertas

Questionários Escalas

Enquete de Opinião

Pesquisa Eleitoral

Pesquisa de Um Sujeito

Observação

Censo Demográfico

Baixo

Pesquisa Acidenta

Baixo

Representatividade na População

Alto

FONTE: Salvador A. M. Sandoval, 2018.

O Diagrama 2 ilustra a complexidade da escolha do método ou métodos de pesquisa que investigadores se defrontam, quando envolve a dupla problemática de aprofundamento do conhecimento e a representatividade dos achados dentro 
de uma população. Em termos gerais, a pesquisa acadêmica no Brasil tem privilegiado as propostas de aprofundamento de conhecimento e ignorado a questão da representatividade desse conhecimento, enquanto que o conhecimento para subsidiar formulação de politica publica exige também uma aproximação em termos de representatividade.

Muitas áreas da pesquisa acadêmica, também, correspondem a campos de políticas públicas: educação, psicologia e psicologia social, política, etc. No Brasil, progressivamente esses espaços de política pública vêm exigindo conhecimentos empíricos com maiores graus de representatividade e os profissionais que atuam nesses campos se defrontam com a necessidade de poder analisar e criticar estudos usando essas metodologias de pesquisa tendo, possivelmente, de planejar pesquisas para subsidiar a formulação de políticas públicas. Apenas as abordagens multimétodos (CRESWELL, 2010) poderão responder à complexidade de análise de fenômenos sociais sob uma perspectiva em seus três níveis.

Pesquisadores atualmente estão diante da necessidade de uma formação ampla em métodos de pesquisa para poderem responder às demandas científicas e de políticas públicas, seja para realizar pesquisas, seja, igualmente importante, para poder avaliar, de modo criterioso, estudos, especialmente quando usados para justificar alguma proposta de política pública. Na ciência moderna, as pesquisas que melhor respondem às exigências para subsidiar os processos de elaboração dessas políticas são aquelas realizadas por meio de abordagens multimétodos.

O Brasil é um dos países que, nos últimos 20 anos, mais se modernizou na área de coleta de dados empíricos a respeito das diversas facetas da sociedade. Portanto, a administração e o gerenciamento das agências que desenvolvem políticas sociais dependem de pesquisas bem fundamentadas metodologicamente que abordem de forma simultânea aspectos individuais e coletivos de segmentos populacionais. Nesse sentido, os gestores hoje precisam ter conhecimento dos múltiplos métodos usados nessas pesquisas, para poder adequadamente encomendar, discutir e avaliar os dados e seus significados na formulação ou implementação de políticas.

Ao mesmo tempo, hoje, os acadêmicos que estudam políticas públicas e políticas de entidades do setor privado têm que ter conhecimento dos vários métodos de pesquisa usados na formulação das políticas e ações de governo, e que são objeto de suas pesquisas, já que a compreensão desses subsídios empíricos é fundamental para desvendar as lógicas que produzem ações e políticas públicas e privadas. Sem conhecimentos de multimétodos, a pesquisa acadêmica atualmente será prejudicada no seu potencial analítico e em sua contribuição relativa ao conhecimento da realidade social. Por esses motivos, torna-se necessária uma formação ao nível de pós-graduação sobre enfoques multimétodos para acompanhar as mudanças na pesquisa social na atualidade. 


\section{REFERÊNCIAS}

BETHLEHEM, J. The Rise of Survey Sampling. Discussion paper (09015), Statistics Netherlands, The Hague/Heerlen, 2009.

BOGARDUS, E. S. Social Distance in the City. Proceedings and Publications of the American Sociological Society, n. 20, p. 40-46, 1926.

CRESWELL, J. W. Projeto de Pesquisa: Métodos Qualitativo, Quantitativo e Misto. Porto Alegre: Artmed, 2010.

ENGELS, F. Condition of the Working Class in England. Nova York: Panther Edition, 1845/1969.

GULLION, J. S. A Brief History of Ethnography. In: Writing Ethnography. Teaching Writing. Rotterdam: Sense Publishers, 2016.

GUTMAN, L.; STOUFFER, S. A.; EDWARD, A.; SUCHMAN, L. C.; DEVINNEY, S.; STAR, A.; WILliAMS JR., R. W. Studies in Social Psychology in World War II: The American Soldier. v. 1. Adjustment During Army Life. Princeton University Press: Princeton, 1949.

LePLAY, F. Les ouvriers europeens. Tours: Alfred Mame et Fils, 1855.

LIKERT, R. A Technique for the Measurement of Attitudes. Archives of Psychology, n.140, p. 1-55, 1932.

NEW INTERNATIONAL. v. 4, n. 12, p. 379-381, dezembro 1938.

OSGOOD, C. E.; SUCI, G.; TANNENBAUM, P. The measurement of meaning. Urbana, IL: University of Illinois Press, 1957.

THOMAS, W.; ZNANIECKI, F. The Polish Peasant in Europe and America. Chicago: University of Illinois Press, 1966.

THURSTONE, L. L. Attitudes can be measured. American Journal of Sociology, n. 33, p.529-54, 1928.

VERMEULEN, H. Early History of Ethnograph and Ethnolog in the German Enlightenment: Anthropological Discourse in Europe and Asia, 1710-1808. Leiden: Privately published, 2008.

WEBER, M. A ciência como vocação. In: WEBER, M. Três tipos de poder e outros escritos. Lisboa: Tribuna da História, 2005.

Texto recebido em 17 de maio de 2018. Texto aprovado em 17 de julho de 2018. 